\title{
Fuzzy modeling and compensation of scale factor for MEMS gyroscope
}

\author{
Li Jianli*, Du Min**, Fang Jiancheng*** \\ *Beijing University of Aeronautics and Astronautics, Beijing, China, E-mail: lijianli@buaa.edu.cn \\ **Institute of Disaster-prevention, Shanhe, Hebei, China, E-mail: dumin@fzxy.edu.cn \\ ***Beijing University of Aeronautics and Astronautics, Beijing, China, E-mail: fangjiancheng@buaa.edu.cn \\ crossref http://dx.doi.org/10.5755/j01.mech.17.4.571
}

\section{Introduction}

The MEMS gyroscopes, an alternative to the conventional rate gyroscope based on a high speed rotor supported in gimbals, can be used to measure the spacecraft angular rate with respect to an inertial reference frame [1, 2]. The benefits of MEMS gyroscope compared to conventional gyroscopes are well known and include robustness, low power consumption, potential for miniature dimensions and hence, for low cost [3, 4]. Despite the potential of the MEMS gyroscope over conventional rate gyroscope, its performance is degraded due to significant scale factor error, misalignment, noise, and temperature bias drift [5]. To decrease the error and improve the performance of MEMS gyroscopes, the misalignment and scale factor errors have been considered [6]. The noise and bias drift have been reported in $[7,8]$, respectively. In these disturbances, the scale factor error is the main error in dynamic maneuvering [9]. The typical output characteristic of MEMS gyroscope include offset, nonlinear and asymmetry error of scale factor $[6,10]$. The error of scale factor is severe and therefore vital to analyze and compensate the scale factor error to improve the performance of the gyroscope to an appropriate level [11].The error of scale factor has been investigated and mainly compensated by employing the 1 th order curve fitting in least squares [12]. However, the approach can not eliminate the nonlinear and asymmetrical error of scale factor. Moreover, the compensation methods including the polynomial curve fitting and segmented et al. are presented respectively [13]. However, these methods are currently faulty in either performance or CPU efficiency.

In this paper, our attention is focused on the modeling and compensation of the scale factor errors of DG (Dual Gimbaled) MEMS gyroscope, which originate from a number of adverse sources including manufacturing tolerance, material inhomogeneity and inevitable mechanical characteristic variation of sensor with rotational rate. Based on the operational principle of the MEMS gyroscope, the physical origin of offset, nonlinear and asymmetry error of scale factor are analyzed. The model of scale factor is proposed. Motivated by the capability of fuzzy logic in managing nonlinearity [14], the error compensation scheme of scale factor is represented by the fuzzy model via experimental data and is online compensated by fuzzy logic. The main idea of this approach is to derive several piecewise linear models for some intervals of rotational rate by using a linear interpolation process. The fuzzy model is saved into memory and used to compensate for the dynamic error of scale factor.

\section{Operating principle of DG MEMS gyroscope}

The DG MEMS gyroscope that had been developed in inertial science was first designed by Draper Lab in 1988. The device is a monolithic silicon gyroscope and consists of a vibrating mass, electrostatic drive electrodes, electrostatic pick-off electrodes, two anchors, supporting beams, inner gimbal and outer gimbal as shown in Fig. 1. The operating principle of DG-MEMS gyroscope is wellunderstood, but a brief summary is given in Fig. 2 .

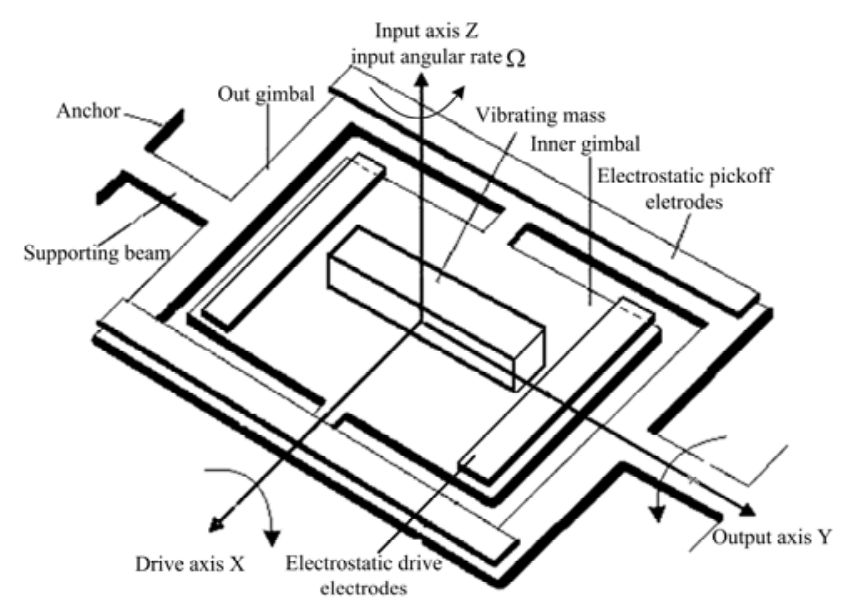

Fig. 1 Schematic structure of DG-MEMS gyroscope

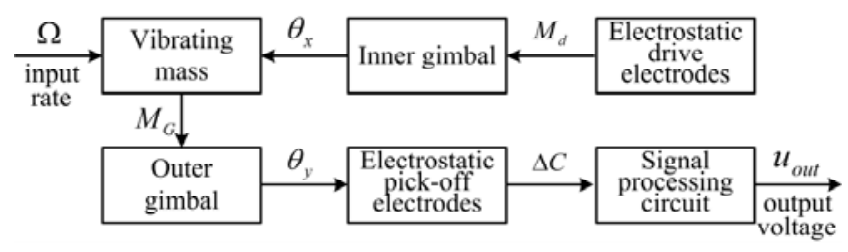

Fig. 2 Operating principle of DG-MEMS gyroscope

The device is a two-gimbal structure supported by supporting beams. In operation, the inner gimbal is driven to vibrate at constant amplitude $\left(\theta_{x}\right)$ by electrostatic torque using electrostatic drive electrodes placed in close proximity. In the presence of an rotational rate $(\Omega)$ normal to the plane of the device, the Coriolis force will cause the outer gimbal to oscillate about its output axis with a frequency equal to the drive frequency and with an amplitude $\left(\theta_{y}\right)$ proportional to the input rotational rate $(\Omega)$. Maximum response is obtained when the inner gimbal is driven at the resonant frequency of the outer gimbal. The readout of device is accomplished by sensing the capacitance difference $\Delta C$ ) between the outer gimbal and a pair of electrostatic pickoff electrodes. When operated open loop, the torsion displacement of the outer gimbal about the output axis is proportional to the input rotational rate $(\Omega)$. A 
detailed description of this device may be found in [15].

\section{Errors analysis and compensation of scale factor}

When gyroscope rotates (externally input rate, $\Omega$ ) in input axis, the vibrating mass experiences a Coriolis inertial torque (MG). The MG can be expressed as

$$
M_{G}=I_{1} \dot{\theta}_{x} \Omega
$$

where $I_{1}$ is the equivalent moment of inertia, $I_{1}=I_{x}+I_{y}-I_{z}, I_{\mathrm{i}}$ is the moment of inertia in $\mathrm{i}$ axis, $i=x$, $y, z$. In accordance with Laplace transform principle, the movement of outer gimbal of DG MEMS gyroscope subjected to MG is described by the equation

$$
\begin{gathered}
\theta_{y}=\frac{M_{G}}{I_{y} S^{2}+D_{y} S+K_{y}}=\frac{I_{1} S \theta_{x}}{I_{y}\left(S^{2}+2 \zeta_{y} \omega_{y} S+\omega_{y}^{2}\right)} \Omega= \\
=\frac{I_{y} \omega_{y}^{2} \sqrt{\left[1-\left(\omega_{x} / \omega_{y}\right)^{2}\right]^{2}+\left(2 \zeta_{y} \omega_{x} / \omega_{y}\right)^{2}}}{{ }^{2}} \Omega
\end{gathered}
$$

where $\theta_{y}$ is torsion amplitude in output axis in engineering unit $(\mathrm{rad}), D_{y}$ and $\zeta_{y}$ are the damping coefficient and ratio, $\zeta_{y}=D_{y} / 2 \sqrt{K_{y} I_{y}}, \omega_{x}$ and $\omega_{y}$ are the resonant radial frequency of drive and output axis, $K_{y}$ is the rigidity of output axis, $K_{y}=\omega_{y}^{2} I_{y}$.

For DG MEMS gyroscope, the electrostatic pickoff electrodes sense torsion amplitude $\left(\theta_{y}\right)$ in output axis and output the capacitance difference ( $\Delta C$ ) between the two electrostatic pickoff electrodes. The capacitance difference resulted from $\theta_{y}$ can been given as

$$
\begin{aligned}
& \Delta C=f\left(\theta_{y}\right)= \\
& =f\left(\frac{I_{1} \omega_{x} \theta_{x} \angle 90}{I_{y} \omega_{y}^{2} \sqrt{\left[1-\left(\omega_{x} / \omega_{y}\right)^{2}\right]^{2}+\left(2 \zeta_{y} \omega_{x} / \omega_{y}\right)^{2}}} \Omega\right)
\end{aligned}
$$

where $f()$ is a function between $\Delta C$ and $\theta_{y}$. The readout of device is accomplished by sensing $\Delta C$ and output voltage processed by signal processing circuit can be written as

$$
\begin{aligned}
& u_{\text {out }}=e(\Delta C)= \\
& =e\left(f\left(\frac{I_{1} \omega_{x} \theta_{x} \angle 90}{I_{y} \omega_{y}^{2} \sqrt{\left[1-\left(\omega_{x} / \omega_{y}\right)^{2}\right]^{2}+\left(2 \zeta_{y} \omega_{x} / \omega_{y}\right)^{2}}} \Omega\right)\right)
\end{aligned}
$$

where $e()$ is a function between output voltage and capacitance difference $(\Delta C)$. The output voltage in engineering unit $(V), u_{\text {out }}$, is proportional to the input rate. The scale factor of the sensor that relates the output voltage to the external input rotation rate $(\Omega)$ is given by

$$
\begin{aligned}
S & =\frac{e\left(f\left(\theta_{y}\right)\right)}{\Omega}= \\
& e\left(f\left(\frac{\Omega I_{1} \omega_{x} \theta_{x} \angle 90}{I_{y} \omega_{y}^{2} \sqrt{\left[1-\left(\omega_{x} / \omega_{y}\right)^{2}\right]^{2}+\left(2 \zeta_{y} \omega_{x} / \omega_{y}\right)^{2}}}\right)\right) \frac{1}{\Omega}
\end{aligned}
$$

The manufacturing tolerance, material inhomogeneity will result in variation the resonant radial frequencies, $\Delta \omega_{x}$ in drive axis and $\Delta \omega_{y}$ in output axis, and mismatch in two oscillating modes of DG MEMS gyroscope. The air damping force on a microstructure reduces and the quality factor, $Q$, increases significantly in rare air with the pressure drop. These inevitable mechanical characteristic variations of sensor are primary factors of offset of scale factor. Moreover, heat results in a decrease in Young's modulus $(E)$, and an increase in compressive thermal internal stress $(\sigma)$. The two effects help to actively lower the resonant frequencies and result in the frequencies mismatch of two oscillating modes too. It is unavoidable that the scale factor thermal offset with heating.

In addition, the distortion and internal stressing effects of structure result in mechanical asymmetrical error of scale factor. The error of demodulation circuit will result in electrical asymmetrical error of scale factor, too. Compared with the conventional rate gyroscope, the electromechanical asymmetrical error of scale factor of DG MEMS gyroscope is significant. The magnitude of asymmetrical error of scale factor is about $0.1 \%$ and can not been neglected in compensation of scale factor errors. The capacitances difference of the DG MEMS gyroscope, as a function of torsion displacement, $\theta_{y}$, are given

$$
\Delta C=f\left(\theta_{y}\right)=-\frac{\varepsilon h_{p}}{\theta_{y}} \ln \frac{z_{0}^{2}-(l+b)^{2} \theta_{y}^{2}}{z_{0}^{2}-\left(l \theta_{y}\right)^{2}}
$$

where $\varepsilon$ is the permittivity of free space, $h_{p}$ is the length of electrostatic pick-off electrode, $z_{0}$ is the original space between drive electrode and inner gimbal, $l$ is the space between inner edge of electrostatic pick-off electrode and $y$ axis, $b$ is the width of electrostatic pick-off electrode. Since $\Delta C$ is nonlinear relative to $\theta_{y}$, scale factor includes nonlinear error. Equation (6) can be simplified

$$
\Delta C \approx P\left(\theta_{y}\right)=\frac{\varepsilon h_{p} b(2 s+b)}{z_{0}^{2}} \theta_{y}
$$

where $P()$ is a simplified linear function between between $\Delta C$ and $\theta_{y}$. According to the (6) and (7), the nonlinear error of scale factor can be expressed as following equation

$$
R_{c}=e\left(f\left(\theta_{y}\right)-P\left(\theta_{y}\right)\right)=e\left(\frac{1}{2} f^{\prime \prime}(\zeta) \theta_{y}^{2}\right)
$$

where $R_{c}$ is nonlinear error in engineering unit $(V)$, $\zeta \in\left(0, \theta_{y}\right)$. Moreover, the micro structure effect and other inevitable adverse sources contribute to the nonlinear error, too. An integrated expression of scale factor can be written as 


$$
S(\Omega)=S_{t}+S_{D}+S_{n}+\varepsilon_{S}
$$

where $S_{t}$ is the nominal scale factor, $S_{D}$ is offset of scale factor, $S_{n}$ is nonlinear and asymmetrical error of scale factor, $\varepsilon_{S}$ is random error of scale factor. It is well known that nonlinear global model can be approximated by a set of piecewise linear local models. The main idea of this fuzzy model is to derive several piecewise linear models for some intervals of rotational rate by using a linear interpolation process over the entire operating rotational rate. The fuzzy model is described by IF-THEN rules, which represent local linear input-output relations of nonlinear scale factor, and is saved into memory and used to compensate for the errors of scale factor. The fuzzy model with n fuzzy rules is described as

$$
S(\Omega)=\left\{\begin{array}{l}
S(0)+\frac{S\left(\Omega_{1}\right)-S(0)}{h} \Omega, 0 \leq \Omega<\Omega_{1} \\
S(0)+\frac{S(0)-S\left(\Omega_{-1}\right)}{h} \Omega, \Omega_{-1}<\Omega<0 \\
S\left(\Omega_{1}\right)+\frac{S\left(\Omega_{2}\right)-S\left(\Omega_{1}\right)}{h}\left(\Omega-\Omega_{1}\right), \Omega_{1} \leq \Omega<\Omega_{2} \\
S\left(\Omega_{-1}\right)+\frac{S\left(\Omega_{-1}\right)-S\left(\Omega_{-2}\right)}{h}\left(\Omega-\Omega_{-1}\right), \Omega_{-2}<\Omega \leq \Omega_{-1} \\
\vdots \\
S\left(\Omega_{n-1}\right)+\frac{S\left(\Omega_{n}\right)-S\left(\Omega_{n-1}\right)}{h}\left(\Omega-\Omega_{n-1}\right), \Omega_{n-1}<\Omega \leq \Omega_{n} \\
S\left(\Omega_{1-n}\right)+\frac{S\left(\Omega_{1-n}\right)-S\left(\Omega_{-n}\right)}{h}\left(\Omega-\Omega_{1-n}\right), \Omega_{-n}<\Omega \leq \Omega_{1-n}
\end{array}\right.
$$

Where $\mathrm{n}$ is the number of rules, $\Omega_{\mathrm{i}}$ and $\mathrm{S}\left(\Omega_{i}\right)$ are the ith externally input rotational rate and scale factor of the ith rotational rate point, $h$ is the density of intervals. The final rotational rate compensated by fuzzy logic, can be written as

$$
\Omega=u_{\text {out }} / S(\Omega)
$$

\section{Experimental results and discussions}

The experiment equipment mainly includes a three-axis rate table, data collect and process system. To decrease temperature noise, the gyroscope is calibrated in a stability temperature. Since the temperature variety is little (about $1 \mathrm{~K}$ ), the scale factor thermal offset can be neglected in the experiment. Moreover, a temperature sensor is used to estimate and compensate bias thermal drift of gyroscope. Testing has shown that the temperature sensor gives a temperature repetition of about $\pm 0.1 \mathrm{~K}$.

The gyroscope is mounted in the fixture such that the nominal input axis is aligned parallel with the spin axis of the rate table. The rate ramped up from zero to the positive constant rotational rate, then bake to negative constant rotational rate, followed by step by step ramp up to maximum rate with specifically rate step. Concretely, the static outputs of gyroscope in two symmetrical orientations are saved respectively. Then, the rate table rotated with 10 rates which including $20^{\circ} / \mathrm{s},-20^{\circ} / \mathrm{s}, 40^{\circ} / \mathrm{s},-40^{\circ} / \mathrm{s}$, $60^{\circ} / \mathrm{s},-60^{\circ} / \mathrm{s}, 80^{\circ} / \mathrm{s},-80^{\circ} / \mathrm{s}, 100^{\circ} / \mathrm{s}$ and $-100^{\circ} / \mathrm{s}$ along the spin axis of the rate table. Every rate is keep for 2 minutes approximately. The gyroscope has power on during the entire calibration process. To every rotational rate, the data is colleted respectively after the rate up to stabilization. The rotational acceleration used in the ramp profile is $5 \mathrm{deg} / \mathrm{sec}^{2}$ in spin axis of the rate table. The rate is ramped up with a max value of $\pm 100 \mathrm{deg} / \mathrm{sec}$, in order to utilize as much as possible of the dynamic range of the gyroscope. The output of gyroscope in any rate is saved into memory as the data of the $i$ th rotational rate.

The date are later read into a MATLAB program for pre-processing (rotational rate and temperature converters, temperature drift compensated) and afterwards calibration. Since the operating temperature range of the gyroscope is little, scale factor temperature sensitivity compensation is not implemented. Based on these data in memory, the scale factors of gyroscope in all rotational rate points are calibrated. The fuzzy model of scale factor can be calibrated by employing the proposed piecewise linear fuzzy rules and the results are shown as Fig. 3. In addition, to compare with other conventional techniques, the 1th order curve fitting and segmented schemes are calibrated and their results are shown in Fig. 3, too. From these results, it can be seen that, the proposed fuzzy model is able to eliminate the offset, nonlinear and asymmetry error of scale factor and more approach to the practical scale factor compared with other conventional methods.

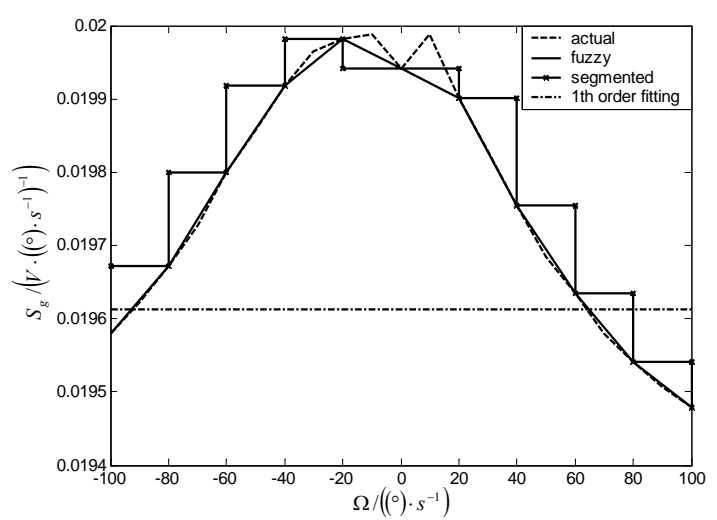

Fig. 3 Calibrated results of scale factor for different models

The second experiment is to check for the performance compensated by proposed fuzzy logic. The rate table rotated with another ten rates including $10 \%, \mathrm{~s},-10 \% \mathrm{~s}$, $30 \% \mathrm{~s},-30 \% \mathrm{~s}, 50 \% \mathrm{~s},-50 \% \mathrm{~s}, 70 \% \mathrm{~s},-70 \% \mathrm{~s}, 90 \% \mathrm{~s},-90 \% \mathrm{~s}$. The 
raw error of gyroscope, $E_{y}$, is shown as Fig. 4. To compensate the dynamic error of scale factor, the proposed fuzzy compensation method is implemented and the error, $E_{c}$, is shown in Fig. 4. In addition, to compare with other compensation technique, the 1 th order curve fitting in a least squares and segmented schemes are employed and the error, $E_{n}$, compensated by 1 th order curve fitting and the error, $E_{f}$, compensated by segmented scheme are shown in Fig. 4 respectively.

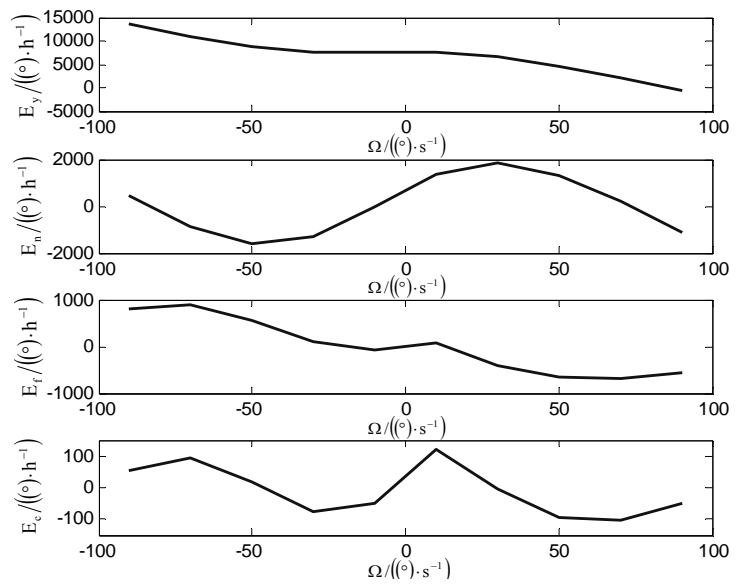

Fig. 4 Raw and compensated errors of scale factor

From these results, it can be seen that, the raw error of gyroscope output, $E_{y}$, is $4053.2^{\circ} / h(1 \sigma)$. The error compensated by proposed fuzzy logic, $E_{c}$, is improved to $79.0^{\circ} / h(1 \sigma)$. Compared with the conventional methods of 1 th order curve fitting and segmented methods, the precision of gyroscope compensated by fuzzy logic is improved 15.4 and 7.5 times respectively.

\section{Conclusions}

In this paper, we analyzed the physical origin of offset, nonlinear and asymmetry error of scale factor for DG MEMS gyroscope. Motivated by the capability of fuzzy logic in managing nonlinear error, the fuzzy compensation was proposed to derive several piecewise linear models for some intervals of rotational rate by using a linear interpolation process. The experimental results showed that the scale factor error is one of the main dynamic adverse resources, and the proposed fuzzy compensation is able to compensate the offset, nonlinear and asymmetry errors of scale factor throughout the entire dynamic range. The results validated the veracity and practicability of the proposed compensation method. The proposed fuzzy compensation outperforms any of its constituent linear counterparts since nonlinear aspects of the model have been taken into consideration. Moreover, this fuzzy compensation outperforms segmented compensation method in same density of intervals. By doing this, we can guarantee a robust performance of device in dynamic maneuvering.

\section{Acknowledgments}

The work was supported by Major State Basic Research Development Program (973 program No.2009CB724000); National High Technology Research and Development Program of China (863 program No.2008AA121302); National Natural Science Foundation of China (60904093,60736025); National science Fund for Distinguished Young Scholars (60825305).

\section{References}

1. Ashwin, A.S. 2002. Integrated micromechanical resonant sensor for inertial measurement system, Berkeley: School of Electrical Engineering and Computer Science, University of California, Berkeley.140.

2. Stan, S.D.; Balan, R.; Maties, V. 2008. Modelling, design and control of 3DOF medical parallel robot, Mechanika 6(74): 68-71.

3. Li Jianli; Fang Jiancheng;Yu Wenbo. 2005. Silicon micromechanical tuning output gyroscope applied at navigation of micro unmanned aerial vehicle, AIAA Guidance, Navigation, and Control Conference and Exhibit,San Francisco, California. 1-8.

4. Li Jianli;Fang Jiancheng;Dong Haifeng. 2010. Structure design and fabrication of a novel dual-mass resonant output micromechanical gyroscope, Journal of Microsystem Technologies 16(2010): 543-552.

5. Alexey, N.;Kazakin; et al. 2003. The impact of technology errors on the operability of the micromechanical gyroscope. 4th Siberian Russian workshop and turorials EDM'2003, Erlagol, Russian. 49-51.

6. Harish, K.; Joglekar; Venka, A. 2004. Gyro scalefactor error and misalignment estimation for a spacecraft, AIAA/AAS, Astrodynamics Specialist Conference and Exhibit, Rhode Island, USA. 16-19.

7. Barakauskas, A.; Kasparaitis, A.; Kausinis, S.; Lazdinas, R. 2009. Analysis of estimation and compensation of angular errors of linear motion, Mechanika 5(79): 52-58.

8. Fang Jiancheng; Li Jianli. 2009. Integrated model and compensation of thermal errors of silicon microelectromechanical gyroscope, IEEE Transactions on Instrumentation and Measurement 58(9): 2923-2930.

9. Liu Qin. et al. 2002. Error analysis and compensation of strapdown inertial navigation system, Journal of Beijing Institute of Technology 11 (2): 117-120.

10. Davis, W.O.D. 2001. Mechanical analysis and design of vibratory micromachined gyroscopes, University of California, Berkeley. 156.

11. Jing Yang. et al. 2004. Analysis and compensation of error in the input device based on inertial sensors, ITCC. Michigan, USA. 790-796.

12. Sungsu, P. et al. 2005. A scheme for improving the performance of a gyroscope-free inertial measurement unit, Sensors and Actuators A(121): 410-420.

13. Lauro, O. et al. 2000. Precision calibration of fiberoptics gyroscopes for mobile robot navigation, IEEE ICRA'00, San Francisco, USA. 2064-2069.

14. Hong, S.K.; Langari, R. 1999. Fuzzy modeling and control of a nonlinear magnetic bearing system, Journal of Intelligent and Fuzzy Systems (4): 335-346.

15. Greiff, P. et al. 1991. Silicon monolithic micromechanical gyroscope, Solid-State Sensors and Actuators, Tansducer'91: 966-968. 
Li Jianli, Du Min, Fang Jiancheng

MEMS GIROSKOPŲ NERAIŠKUSIS MODELIAVIMAS IR MASTELIO FAKTORIAUS IVERTINIMAS

\section{R e z i u m è}

MEMS giroskopu mastelio faktoriaus paklaidos būna didelès ir dèl to gyvybiškai svarbu jas analizuoti ir kompensuoti gerinant giroskopu charakteristikas. Remiantis giroskopo „Dual Gimbaled MEMS“ veikimo principu, analizuojama fizinè paklaidų kilmè, mastelio faktoriaus netiesiškumo ir asimetrijos paklaidos. Pasiūlytas mastelio faktoriaus paklaidų modelis. Pateiktas neraiškiosios logikos mastelio faktoriaus paklaidų kompensavimo modelis. Bandymu rezultatai rodo, kad dinaminès giroskopo paklaidos yra 4053,2 $/ h(1 \sigma)$. Iprastiniais ir daliniais metodais lyginant pirmos eilès kreivių sutapima, giroskopo tikslumas, kompensuojamas neraiškiaja logika, pagerèja atitinkamai 15.4 ir 7.5 karto. Tai patvirtina neraiškiojo modelio tikslumą ir pagerina giroskopo dinaminị manevringumą.
Li Jianli, Du Min, Fang Jiancheng

\section{FUZZY MODELING AND COMPENSATION OF SCALE FACTOR FOR MEMS GYROSCOPE}

\section{S u m m a r y}

The scale factor error of MEMS gyroscope is severe and therefore vital to analyze and compensate the error to improve the performance of gyroscope. Based on the operational principle of Dual Gimbaled MEMS gyroscope, it is analyzed that the physical origin of offset, nonlinear and asymmetry errors of scale factor. The error model of scale factor is proposed. The fuzzy logic, a method to compensate scale factor, is presented. The experimental results show that the dynamic raw error of gyroscope is $4053.2^{\circ} / h(1 \sigma)$, and error compensated by the fuzzy logic is improved to $79.0^{\circ} / h(1 \sigma)$. Compared with the conventional methods of 1 th order curve fitting and segmented methods, the precision of gyroscope compensated by fuzzy logic is improved 15.4 and 7.5 times respectively. It verifies the accuracy of the fuzzy model and compensation, and improves of gyroscope in dynamic maneuvering.

Received April 07, 2011 Accepted August 23, 2011 